\title{
Cytological Features That Differentiate Follicular Neoplasm from Mimicking Lesions
}

Kanghee Han · Hwa-Jeong Ha Joon Seog Kong · Jung-Soon Kim Jae Kyung Myung ${ }^{1} \cdot$ Jae Soo Koh Sunhoo Park ${ }^{1} \cdot$ Myung-Soon Shin Woo-Tack Song · Hye Sil Seol Seung-Sook Lee

Department of Pathology, Korea Cancer Center Hospital, Korea Institute of Radiological and Medical Sciences (KIRAMS), Seoul; ${ }^{1}$ Laboratory of Radiation Pathology, Korea Institute of Radiological and Medical Sciences (KIRAMS), Seoul, Korea

Received: August 30, 2017

Revised: January 12, 2018

Accepted: January 17, 2018

\section{Corresponding Author}

Seung-Sook Lee, MD, PhD

Laboratory of Radiation Pathology, Korea Cancer Center Hospital, Korea Institute of Radiological and Medical Sciences (KIRAMS), 75 Nowon-ro,

Nowon-gu, Seoul 01812, Korea

Tel: +82-2-970-1268

Fax: +82-2-970-2430

E-mail: sslee@kirams.re.kr
Background: It is difficult to correctly diagnose follicular neoplasms (FNs) on fine-needle aspiration cytology (FNAC) because it shares many cytological features with other mimicking lesions. The aim of this study was to identify the cytological features that differentiate FNs from mimicking lesions. Methods: We included the cytological slides from 116 cases of thyroid FN diagnosed on FNAC, and included their subsequent histological diagnoses. We evaluated the cytological architectural pattern and nuclear features of the lesions according to their histological groups. Results: The final histological diagnoses of the 116 cases varied, and included 51 FNs (44\%), 47 papillary thyroid carcinomas (40\%) including follicular variant, and seventeen cellular nodular hyperplasias (15\%). Regardless of the final histological diagnosis, microfollicular pattern was observed in most cases. On the other hand, trabecular pattern was identified in $34 \%$ of FNs, but not in any other lesions. Additionally, elongated nuclei and ground glass chromatin were found in only some papillary thyroid carcinomas. Conclusions: This study shows that the trabecular pattern is a representative cytological feature of FNs that can be used to distinguish FNs from mimicking lesions. In addition, nuclear shape and chromatin pattern can be used to further confirm the diagnosis of FNs from mimicking lesions through FNAC.

Key Words: Follicular neoplasm; Fine needle aspiration cytology; Differential diagnosis
Fine-needle aspiration cytology (FNAC) is an accurate and useful method for evaluating thyroid lesions and has been adopted as a tool for the diagnosis of thyroid nodules prior to surgery. This convenient tool could be used to reduce the rate of unnecessary treatments, such as surgery, for benign lesions. ${ }^{1,2}$ More rapid and accurate diagnoses will also increase the effectiveness of treatments. ${ }^{3}$ Moreover, because of the recent normalization of regular health checkups, the diagnosis of thyroid lesions has increased and FNAC is used more frequently to identify indeterminate nodules in the thyroid. Although FNAC is an excellent diagnostic tool for identifying thyroid nodules, the accuracy of diagnosis remains an issue in diagnosing follicular neoplasms (FNs) including follicular adenoma (FA), follicular carcinoma (FC), and Hurthle cell carcinoma $(\mathrm{HC})$ and cytologically similar lesions.

In general, FNAC diagnosis of FNs depends on highly cellular aspirate composed of uniform follicular cells arranged in microfollicular pattern and crowded clusters. However, because FNs display cytological features similar to other lesions including cellular nodular hyperplasia $(\mathrm{NH})$, follicular variant of papillary thyroid carcinoma (FVPTC), and classic papillary thyroid carcinoma (PTC), correctly distinguishing FNs from these mimicking lesions through FNAC can be difficult. For these reasons, to correctly diagnose FNs in the thyroid using FNAC is a great challenge in clinical practice. Therefore, in this study, we investigated the cytological features of FNs to clearly distinguish it from other mimicking lesions by comparing features such as cytological architecture and detailed nuclear features, as well as histological diagnosis. 


\section{MATERIALS AND METHODS}

We searched for cytology cases that were diagnosed as or suspected to be FNs through FNAC at the Department of Pathology, Korea Cancer Center Hospital (KCCH, Seoul, South Korea) between January 2010 and June 2015, using the pathology database of $\mathrm{KCCH}$. We selected 116 cases where the thyroid was subsequently removed. The slides were reviewed by two pathologists and one cytotechnician.

Aspirations were performed with a 22-25-gauge needle attached to a $10-\mathrm{mL}$ disposable syringe. The collected samples were placed onto slides, fixed with 95\% ethyl alcohol, and stained with the Papanicolaou stain. Surgically resected tissues were embedded in paraffin, sectioned into 4- $\mu \mathrm{m}$ slices and stained with hematoxylin and eosin (H\&E).

Diagnoses of the 116 cases through FNAC were compared with the histological diagnoses. The cytological analysis focused on both cytomorphological architecture and individual nuclear features. The architectural features evaluated were microfollicular architecture, multilayer rosettes, branching monolayer sheets, syncytial fragments, and trabecular pattern. Nuclear characteristics were analyzed based on chromatin clearing, nuclear grooves, intranuclear inclusions, anisonucleosis, and nuclear shape in each tissue-proven diagnostic group.

\section{Definition of diagnostic terms}

The FNAC cases in this study belong to the diagnostic category IV "FN or suspicious for FN" by the Bethesda system ${ }^{3}$ which refers to a cellular aspirate composed of follicular cells showing significant cell crowding and/or microfollicular pattern without nuclear features of papillary carcinoma. Here, we tried to identify whether the diagnostic category IV by the Bethesda system is correlated with the histological diagnosis of FNs and, if there is a discrepancy, what would be helpful findings to differentiate them. Thus, the term "FN" in this study was used as a word collectively referred to as FA, FC, and HC by histological diagnosis. Therefore, FNs used in this study is different in meaning from the FN used in the Bethesda system.

Here, the term "PTC" refers to a classic papillary thyroid carcinoma having papillary architecture and characteristic nuclear features such as chromatin clearing, nuclear grooves, and intranuclear inclusions. Additionally, in the present study, FVPTCs were subdivided into three subgroups by the histologic examination and diagnostic criteria proposed in previous studies ${ }^{4,5}$ : noninvasive follicular thyroid neoplasm with papillary-like nuclear features (NIFTP), ${ }^{4}$ invasive encapsulated follicular variant of pap- illary thyroid carcinoma (invasive EFVPTC), and infiltrative FVPTC. We examined the difference between the subtypes of FVPTC.

\section{Statistical analysis}

All statistical analyses were performed using the SPSS ver. 18 (SPSS Inc., Chicago, IL, USA). A Pearson's chi-square test was performed to evaluate the relevance of correlation between cytological architecture, nuclear characteristics and categorical variables. For all analyses, a p-value $<.05$ was considered statistically significant.

This study was approved by the Institutional Review Board of Korea Cancer Center Hospital with a waiver of informed consent (IRB number: K-1710-002007).

\section{RESULTS}

\section{Histological correlation}

Table 1 shows the final histological diagnoses for the 116 cases diagnosed as Bethesda diagnostic category IV, "FN or suspicious for FN" using FNAC. The concordance rate between preoperative cytological diagnosis and histological diagnosis of FNs was only $44 \%$ (Table 1). Histological follow-up identified $40 \%$ of the cases diagnosed as FNs by preoperative cytology as FVPTC or PTC. The most commonly misdiagnosed lesion was FVPTC. The detailed results are as follows: 27 cases of FA (23\%), 23 cases of FC (20\%), one case of HC (1\%), 35 cases of FVPTC (30\%), 12 cases of PTC (10\%), 17 cases of NH (15\%), and one case of Hashimoto thyroiditis (HT; 1\%). The representative histological and cytological features of the lesions are shown in Fig. 1.

\section{Evaluation of cytological features}

The aspirates of the lesions were generally cell-rich with an

Table 1. Histological categories of 116 cases diagnosed as Bethesda category IV, "FN or suspicious for FN" through fine-need aspiration cytology

\begin{tabular}{ccc}
\hline & Histological diagnosis & No. of cases (\%) \\
\hline FN & FA & $27(23)$ \\
& FC & $23(20)$ \\
Non-FN & HC & $1(1)$ \\
& FVPTC & $35(30)$ \\
& PTC & $12(10)$ \\
& NH & $17(15)$ \\
& HT & $1(1)$ \\
\hline
\end{tabular}

FN, follicular neoplasm; FA, follicular adenoma; FC, follicular carcinoma; $\mathrm{HC}$, Hurthle cell carcinoma; FVPTC, follicular variant of papillary thyroid carcinoma; PTC, classic papillary thyroid carcinoma; $\mathrm{NH}$, nodular hyperplasia; $\mathrm{HT}$, Hashimoto thyroiditis. 
abundance of follicular cell groups showing microfollicular patterns, syncytial configurations, and/or abundant isolated cells, which was sufficient evidence to suspect FNs in FNAC smears.
To determine cytological features unique to FNs, we evaluated each case in terms of both cytological architecture and nuclear characteristics.
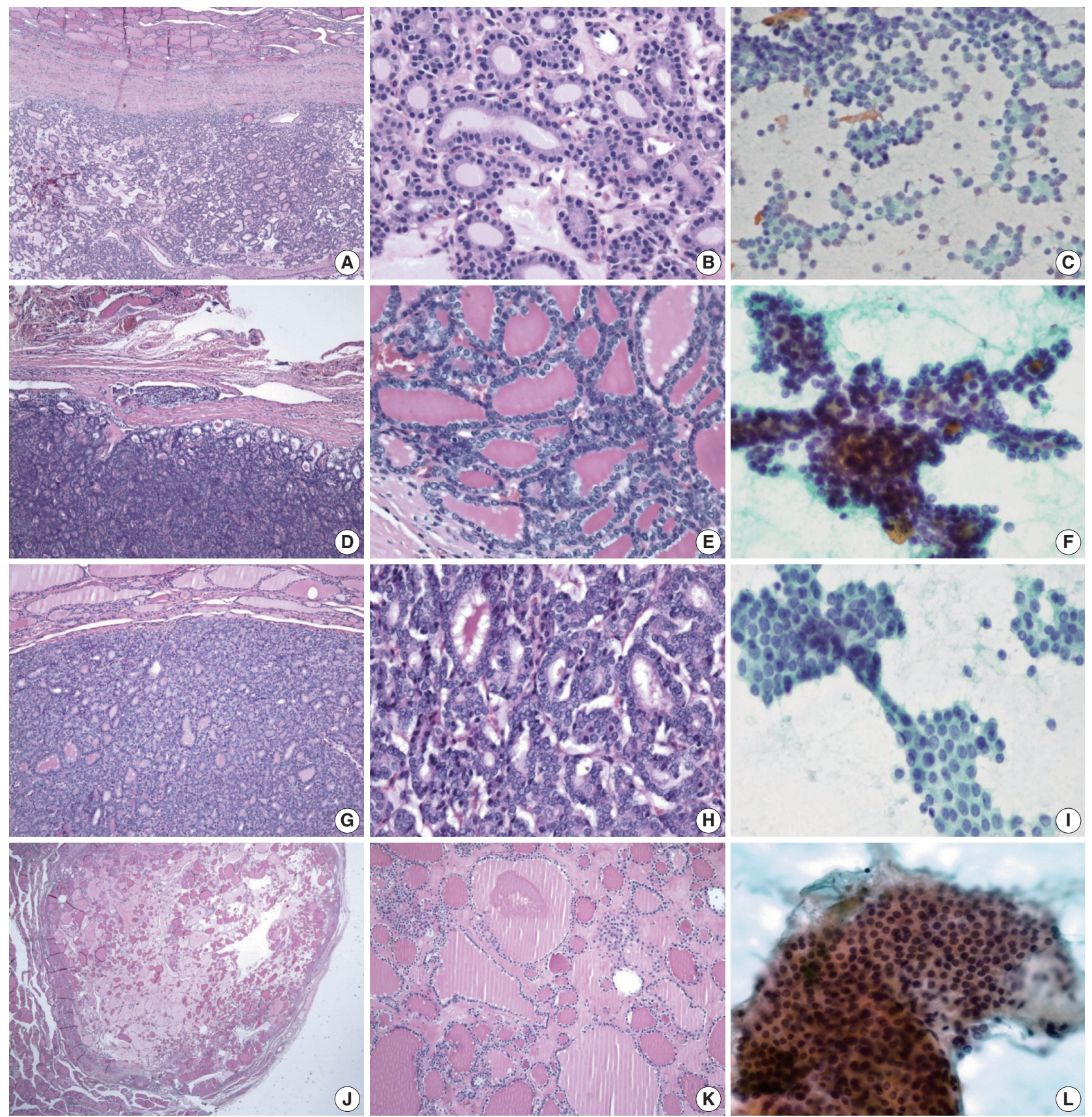

Fig. 1. Histological and cytological features of thyroid neoplasm. (A, B) Histological features of follicular adenoma. (A) The tumor is enclosed in a thick fibrous capsule. (B) The architectural pattern is follicular, and cells are uniform, round and dark. (C) Cytological features of follicular adenoma. Abundant follicular cells are seen, with little colloid. (D, E) Histological features of follicular carcinoma. (D) Vascular invasion in fibrous capsule of follicular carcinoma. (E) Colloid-containing well-formed follicles. (F) Cytological feature of follicular carcinoma. Note that microfollicles show three dimensional branching cellular clusters with trabecular pattern. $(G, H)$ Histological features of follicular variant of papillary carcinoma. (G) The tumor resembles follicular neoplasm. (H) Nuclei are round to ovoid, similar to follicular neoplasm but show slight enlargement and overlapping. (I) Cytoloigcal feature of papillary carcinoma. Monolayer branching sheet of papillary carcinoma is compared with three-dimensional branching cluster of follicular carcinoma. (J, K) Histological features of nodular hyperplasia. Follicles are of varying sizes with abundant colloid. (L) Aspirate of nodular hyperplasia shows flat sheets of uniform follicular cells and colloid. 


\section{Cytological architecture}

We reviewed the cytology smears, focusing on cytological architectures generally regarded as features of FNs such as microfollicular patterns, the trabecular pattern, multilayer rosettes, branch- ing sheets, and syncytial fragments, and analyzed the frequency of these findings in each histologically proven diagnosis (Table 2).

Regardless of the final histological diagnosis, the microfollicular pattern was observed in most cases (Table 2). The microfol-

Table 2. Cytological architecture on the aspirates according to histological diagnosis

\begin{tabular}{lccccc}
\hline \multirow{2}{*}{ Histological diagnosis } & \multicolumn{5}{c}{ Cytological architecture } \\
\cline { 2 - 6 } & Microfollicular pattern & Multilayer rosettes & Trabecular pattern & Branching monolayer sheets & Syncytial fragments \\
\hline FA $(n=27)$ & $27(100)$ & $4(15)$ & $9(33)$ & $3(11)$ & $26(96)$ \\
FC $(n=23)$ & $23(100)$ & $8(35)$ & $8(35)$ & $1(4)$ & $22(96)$ \\
HC $(n=1)$ & $1(100)$ & 0 & 0 & 0 & 0 \\
FVPTC $(n=35)$ & $33(94)$ & $16(46)$ & 0 & $12(34)$ & $30(86)$ \\
PTC $(n=12)$ & $10(83)$ & $5(42)$ & 0 & 0 & $12(100)$ \\
NH $(n=17)$ & $17(100)$ & $1(6)$ & 0 & $3(17)$ & $11(65)$ \\
HT $(n=1)$ & $1(100)$ & 0 & 0 & 0 & $1(100)$ \\
Total $(n=116)$ & $112(97)$ & $34(29)$ & $17(15)$ & $19(16)$ & $101(87)$ \\
\hline
\end{tabular}

Values are presented as number (\%).

FA, follicular adenoma; FC, follicular carcinoma; HC, Hurthle cell carcinoma; FVPTC, follicular variant of papillary thyroid carcinoma; PTC, classic papillary thyroid carcinoma; $\mathrm{NH}$, nodular hyperplasia; $\mathrm{HT}$, Hashimoto thyroiditis.
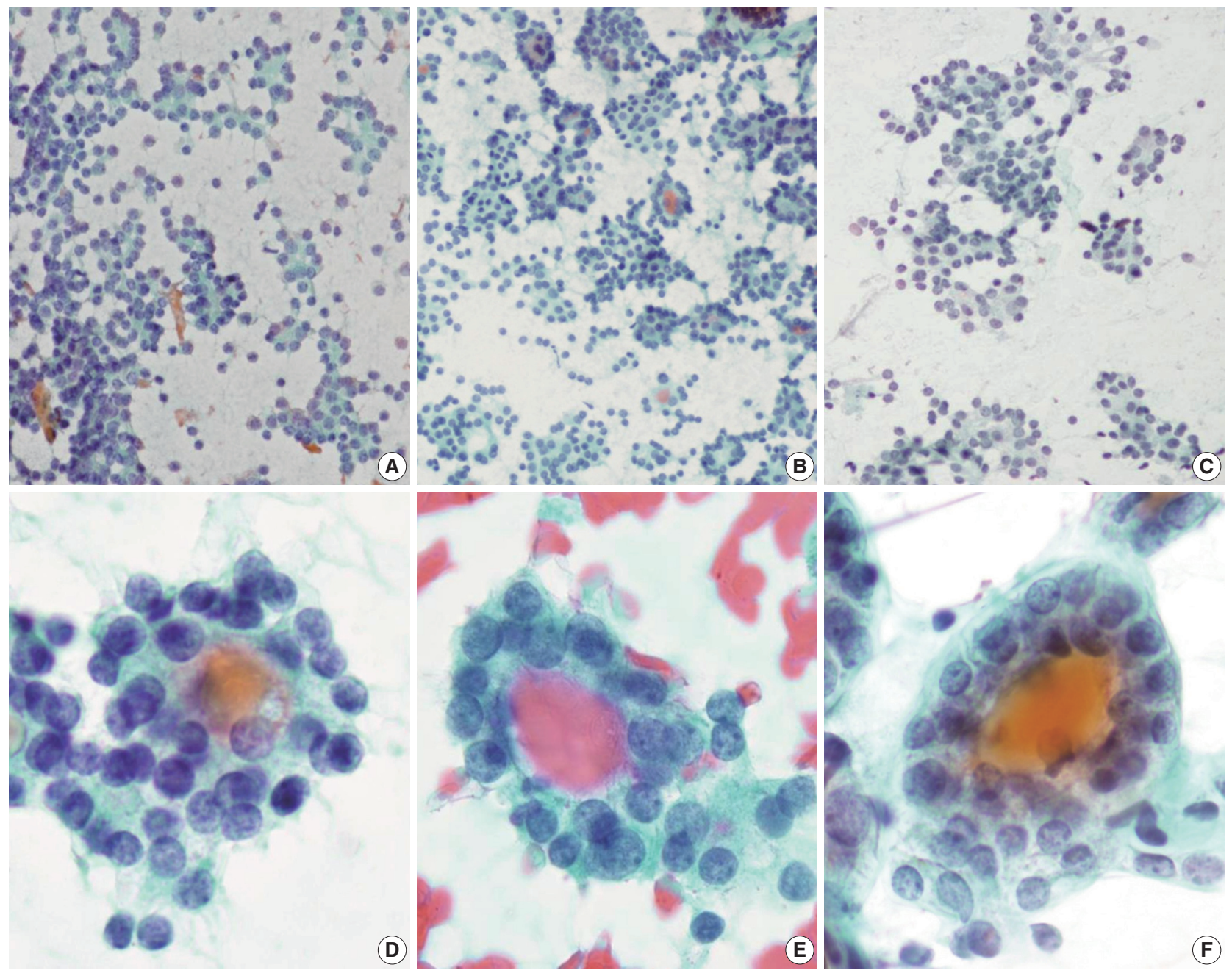

Fig. 2. Microfollicular pattern $(A-C)$ and multilayer rosettes $(D-F)$ are found not only in follicular neoplasm but also in nodular hyperplasia or follicular variant of papillary carcinoma. (A, D) Follicular neoplasm. (B, E) Follicular variant of papillary carcinoma. (C, F) Nodular hyperplasia. 
licular pattern was found not only in FNs, but also in PTC/ FVPTC and NH. Based on this finding, PTC/FVPTC and NH might be mistaken for FNs through FNAC. The observation rates of the microfollicular pattern in each disease entity are as follows: $100 \%$ of FNs cases (FA, FC, and HC), $94 \%$ of FVPTC cases (33/35), 83\% of PTC cases (10/12), 100\% of NH cases, and $100 \%$ of HT cases (Table 2, Fig. 2A-C). These results suggest that the presence of microfollicles is not specific to FNs.
The presence of the multilayer rosette, which is thought to be a representative cytological feature of FNs, was limited to $15 \%$ of FA cases (4/27) and 35\% of FC cases (8/23). Moreover, multilayer rosettes were found more frequently in FVPTC or PTC than in FA or FC ( $46 \%$ of FVPTC cases [16/35] and $42 \%$ of PTC cases [5/12]) in this study (Fig. 2D, E). Multilayer rosettes were also found in cases of $\mathrm{NH}$ (Fig. 2F). Based on these data, the presence of multilayer rosettes in thyroid FNAC smears

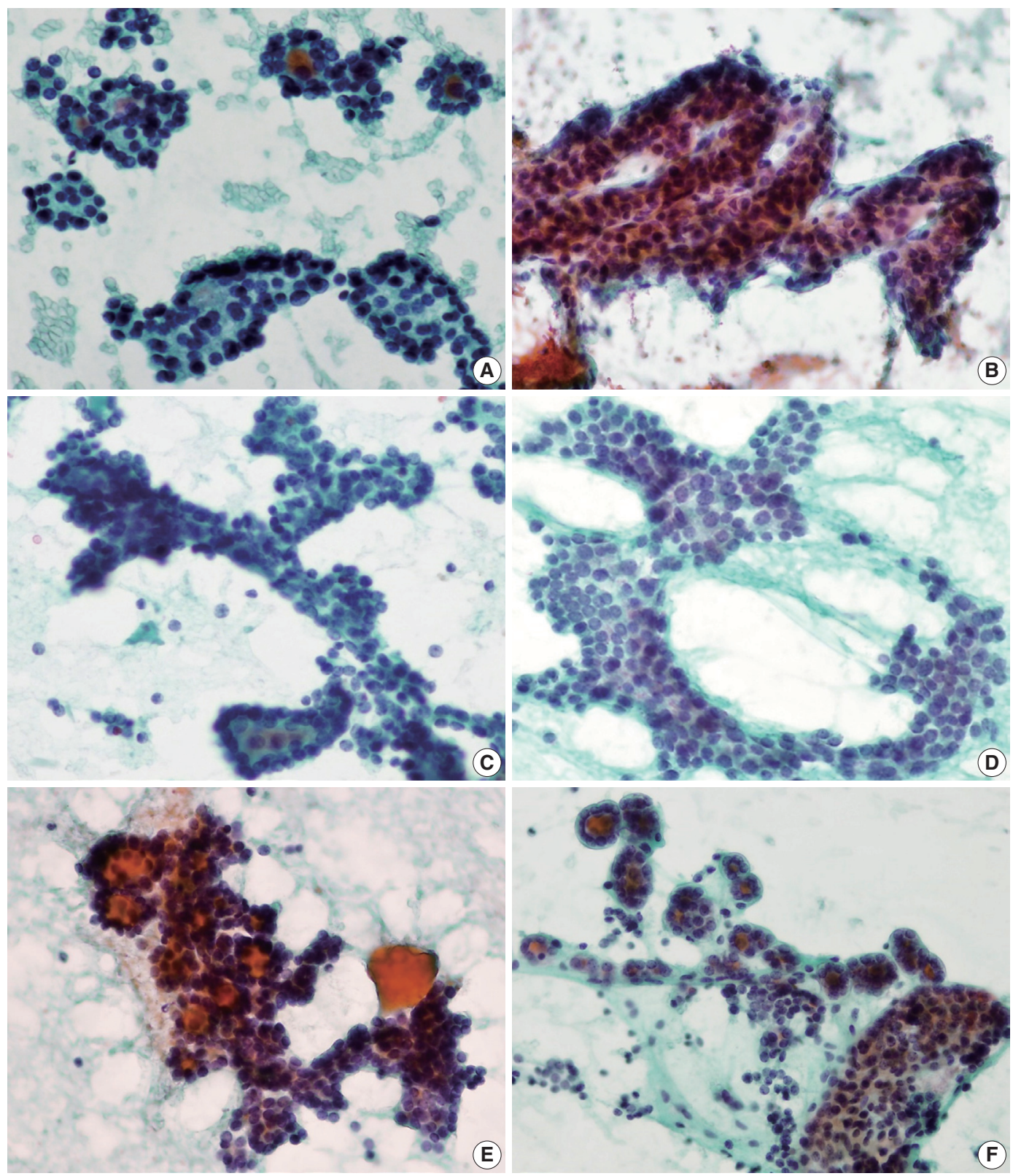

Fig. 3. Different features of cell clusters in follicular neoplasm (FN), follicular variant of papillary thyroid carcinoma (FVPTC), and nodular hyperplasia. (A, B) Trabecular pattern in FN. (C) Branching cell sheets in FN. Branching fragments of FN show ribbon-forming overlapped follicular cells. Note the three-dimensional arrangement of follicular cells of FN (C), which is contrasted with monolayer branching sheet of FVPTC (D). (E) Multilayered cellular ball clusters of FN show branching and trabecular pattern. (F) On the other hand, cell ball clusters in NH show no branching pattern with large non-branching cellular sheet in the colloid background. 
should be considered a feature of both FNs and PTC.

Syncytial cellular fragments were common in FNs, FVPTC, PTC, and NH (Table 2). Interestingly, the trabecular pattern was exclusively observed in FNs (33\% of FA cases and 35\% of FC cases) (Table 2, Fig. 3A-C, E). In FVPTC, branching cellular fragments tended to appear as monolayer sheets; this is in contrast to the cellular overlapping observed in FNs cells (Fig. 3C, D). Branching monolayer sheets were more common in FVPTC (12/35 cases, 34\%) than in FNs (FA [3/27 cases, 11\%], FC [1/23 cases, 4\%], and HC [0/1 case]) (Table 2). Multilayered cellular ball clusters were also observed in $\mathrm{NH}$, but in contrast to $\mathrm{FNs}$, no branching trabecular pattern was seen (Fig. 3E, F).

\section{Cytological nuclear characteristics}

Next, we analyzed and compared the nuclear features of each disease (Table 3). Chromatin clearing was observed in $86 \%$ of FVPTC cases (30/35) and 75\% of PTC cases (9/12). In contrast, much lower frequencies were observed in FNs cases; only $4 \%$ of FA cases (1/27), $17 \%$ of FC cases (4/23), and $0 \%$ of HC cases $(0 / 1)$ displayed chromatin clearing. The nuclei of FNs and $\mathrm{NH}$ were slightly more hyperchromatic than those of PTC or FVPTC, and were found to contain microgranular chromatin (Fig. 4). In addition, nuclear grooves were observed in all histological groups except HT, and their observation rates ranged from $22 \%$ to $100 \%$ (Table 3, Fig. 4). Intranuclear inclusions, another

Table 3. Cytological nuclear characteristics on the aspirates according to histological diagnosis

\begin{tabular}{lcccc}
\hline \multirow{2}{*}{ Histological diagnosis } & \multicolumn{3}{c}{ Nuclear characteristic } \\
\cline { 2 - 5 } & Chromatin clearing & Nuclear groove & Intranuclear cytoplasmic inclusion & Anisonucleosis \\
\hline FA $(n=27)$ & $1(4)$ & $11(41)$ & $2(7)$ & $7(26)$ \\
FC $(n=23)$ & $4(17)$ & $5(22)$ & $3(13)$ & $20(87)$ \\
HC $(n=1)$ & 0 & $1(100)$ & 0 & 0 \\
FVPC $(n=35)$ & $30(86)$ & $32(91)$ & $17(49)$ & $32(91)$ \\
PTC $(n=12)$ & $9(75)$ & $4(33)$ & $2(17)$ & $10(83)$ \\
NH $(n=17)$ & 0 & $8(47)$ & 0 & $4(24)$ \\
HT $(n=1)$ & 0 & 0 & & $1(100)$ \\
\hline
\end{tabular}

Values are presented as number (\%).

FA, follicular adenoma; FC, follicular carcinoma; HC, Hurthle cell carcinoma; FVPTC, follicular variant of papillary thyroid carcinoma; PTC, classic papillary thyroid carcinoma; $\mathrm{NH}$, nodular hyperplasia; $\mathrm{HT}$, Hashimoto thyroiditis.
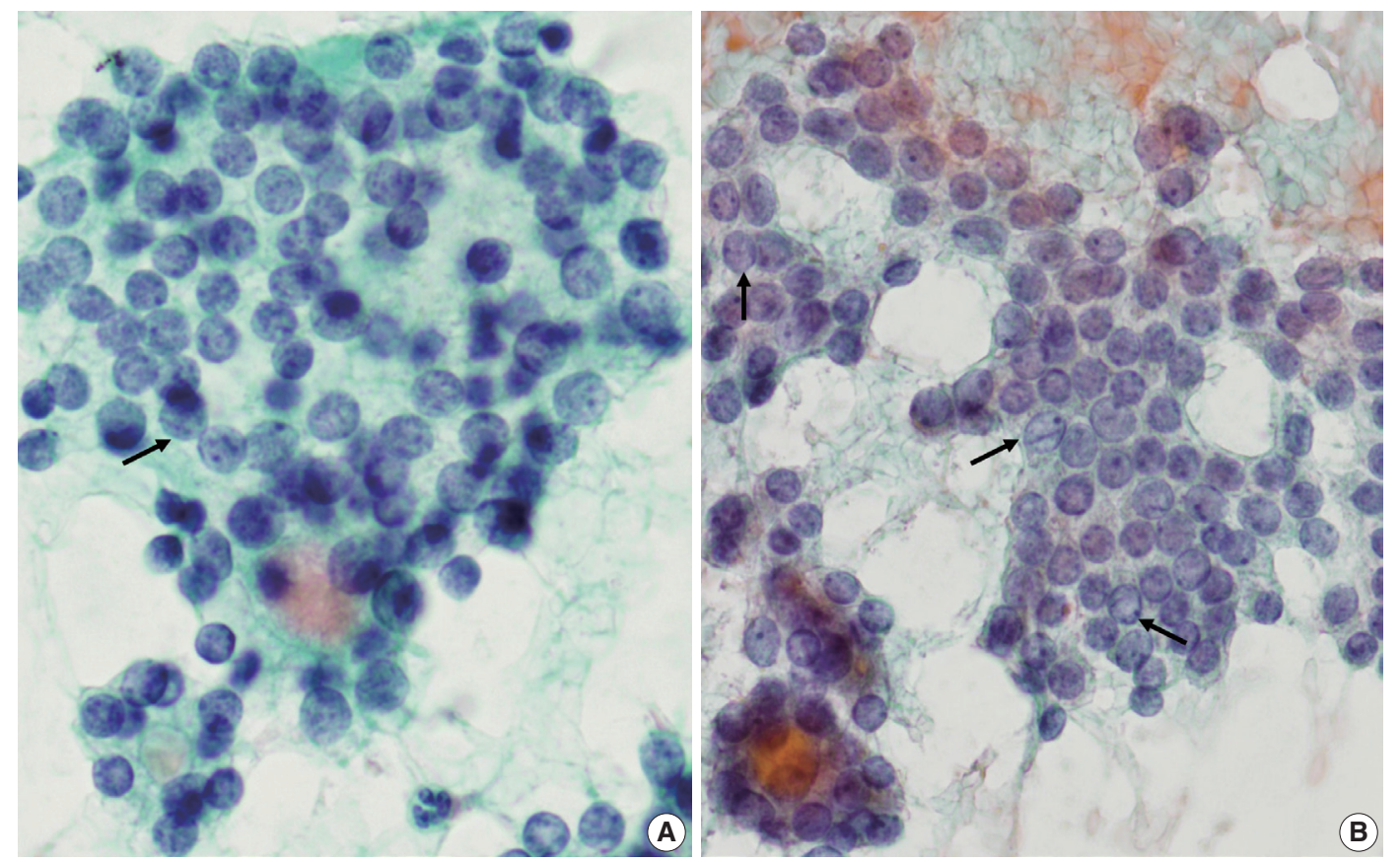

Fig. 4. Nuclear features of follicular neoplasm (FN) and nodular hyperplasia (NH). The nuclear features of FN (A) and NH (B) are similar. Both have round nuclei with microgranular chromatin and nuclear grooves (arrows). However, note the difference that FN (A) shows a three-dimensional arrangement and slightly hyperchromatic nuclei but $\mathrm{NH}(\mathrm{B})$. $\mathrm{NH}$ has monolayer honeycomb sheets of follicular cells that do not overlap. 

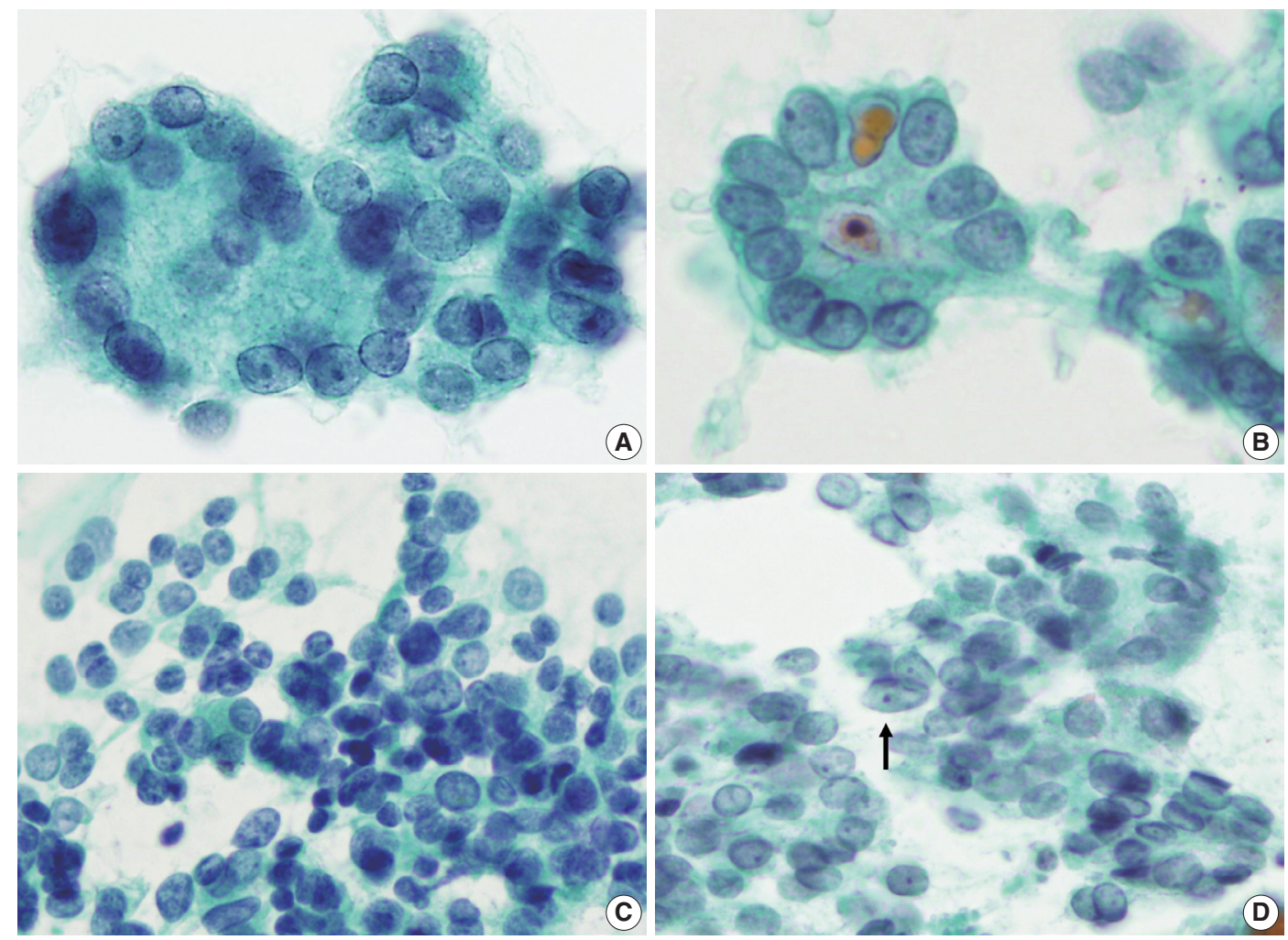

Fig. 5. Nuclear features of follicular neoplasm (FN) and follicular variant of papillary thyroid carcinoma (FVPTC). Please note the subtle difference of nuclear shape and chromatin between follicular carcinoma (A, C) and FVPTC (B, D). (A, C) In FN, nuclei are round to ovoid and chromatin is finely granular rather than ground glass or vesicular pattern. (B, D) Nuclei in FVPTC are slightly elongated (arrow) and have a groundglass appearing chromatin pattern.

nuclear characteristic of papillary carcinomas, were found in $49 \%$ of FVPTC cases (17/35) and $17 \%$ of PTC cases (2/12), as well as in $7 \%, 13 \%$, and $6 \%$ of FA, FC, and $\mathrm{NH}$ cases, respectively.

Regarding nuclear size variation, FVPTC, PTC, and FC showed anisonucleosis in most cases ( $91 \%$ of FVPTC cases [32/35], 83\% of PTC cases [10/12], and $87 \%$ of FC cases [20/23]), except for FA and NH, where it was observed less frequently (26\% [7/27] and 24\% [4/17], respectively). We noticed that, in some cases, the nuclear shape was different for each disease. While approximately half of the FVPTC/PTC cases showed round-to-oval or oval-to-elongated nuclei, most FNs and all NH cases showed round nuclei (Figs. 4, 5). Round-tooval nuclei were commonly observed in FNs and FVPTC/PTC cases, but elongated nuclei were only observed in FVPTC cases (Table 4, Fig. 5). From these observations, a combination of nuclear shape and nuclear chromatin pattern analysis would be useful for differentiating between FNs and PTC/FVPTC through FNAC.

\section{Statistical significance}

Categorizing the cases into FNs and thyroid diseases other
Table 4. Analysis of nuclear shape on the aspirates according to histological diagnosis

\begin{tabular}{lccc}
\hline Histological diagnosis & Round & Round-to-oval & Oval-to-elongated \\
\hline FA $(n=27)$ & $19(70)$ & $8(30)$ & 0 \\
FC $(n=23)$ & $21(91)$ & $2(9)$ & 0 \\
HC $(n=1)$ & $1(100)$ & 0 & 0 \\
FVPTC $(n=35)$ & $15(43)$ & $7(20)$ & $13(37)$ \\
PTC $(n=12)$ & $9(75)$ & $3(25)$ & 0 \\
NH $(n=17)$ & $16(94)$ & $1(6)$ & 0 \\
HT $(n=1)$ & $1(100)$ & 0 & 0 \\
\hline
\end{tabular}

Values are presented as number (\%).

FA, follicular adenoma; FC, follicular carcinoma; HC, Hurthle cell carcinoma; FVPTC, follicular variant of papillary thyroid carcinoma; PTC, classic papillary thyroid carcinoma; $\mathrm{NH}$, nodular hyperplasia; $\mathrm{HT}$, Hashimoto thyroiditis.

than follicular neoplasm (non-FN), the statistical significance of various variables was examined. For the cytological architecture, trabecular pattern $(\mathrm{p}<.001)$ and branching monolayer sheets $(\mathrm{p}=$ .028) were statistically associated with histological diagnosis FN or non-FN, respectively. On the other hand, there was no significant correlation between histological diagnosis and other cytological architectures such as microfollicular pattern, multilayer rosettes and syncytial fragments (Table 5). Regarding nuclear fea- 
Table 5. Differentiation between FN and non-FN

\begin{tabular}{|c|c|c|c|c|}
\hline & $\begin{array}{c}\text { FN } \\
(n=51)\end{array}$ & $\begin{array}{l}\text { Non-FN } \\
(n=65)\end{array}$ & $\chi^{2}$ & $\begin{array}{c}p- \\
\text { value }\end{array}$ \\
\hline \multicolumn{5}{|l|}{ Cytological architecture } \\
\hline Microfollicular pattern & & & & .071 \\
\hline Present & $51(100)$ & $61(94)$ & 3.251 & \\
\hline Absent & 0 & $4(6)$ & & \\
\hline Multilayer rosettes & & & & .226 \\
\hline Present & $12(24)$ & $22(34)$ & 1.468 & \\
\hline Absent & $39(76)$ & $43(66)$ & & \\
\hline Trabecular pattern & & & & $<.001$ \\
\hline Present & $17(33)$ & 0 & 22.037 & \\
\hline Absent & $34(67)$ & $65(100)$ & & \\
\hline \multicolumn{5}{|c|}{ Branching monolayer sheets } \\
\hline Present & $4(8)$ & $15(23)$ & 4.842 & .028 \\
\hline Absent & $47(92)$ & $50(77)$ & & \\
\hline \multicolumn{5}{|l|}{ Syncytial fragments } \\
\hline Present & $48(94)$ & $54(83)$ & 3.283 & .070 \\
\hline Absent & $3(6)$ & $11(17)$ & & \\
\hline \multicolumn{5}{|l|}{ Nuclear characteristics } \\
\hline Chromatin clearing & & & & $<.001$ \\
\hline Present & $5(10)$ & $39(60)$ & 30.584 & \\
\hline Absent & $46(90)$ & $26(40)$ & & \\
\hline Nuclear groove & & & & $<.001$ \\
\hline Present & $17(33)$ & $44(68)$ & 13.531 & \\
\hline Absent & $34(67)$ & $21(32)$ & & \\
\hline \multicolumn{5}{|c|}{ Intranuclear cytoplasmic inclusion } \\
\hline Present & $5(10)$ & $20(32)$ & 7.430 & .006 \\
\hline Absent & $46(90)$ & $45(68)$ & & \\
\hline Anisonucleosis & & & & .048 \\
\hline Present & 27 (53) & $46(71)$ & 3.894 & \\
\hline Absent & $24(47)$ & $19(29)$ & & \\
\hline
\end{tabular}

Values are presented as number (\%).

FN, follicular neoplasm; Non-FN, disease other than FN.

tures, chromatin clearing $(\mathrm{p}<.001)$, nuclear groove $(\mathrm{p}<.001)$, intranuclear cytoplasmic inclusion $(\mathrm{p}=.006)$ and anisonucleosis $(\mathrm{p}=.048)$ were statistically associated with non-FN.

\section{Cytological architecture and nuclear characteristics of subtypes of FVPTC}

We subdivided FVPTC cases into three subtypes: NIFTP, invasive EFVPTC, and infiltrative FVPTC. Of the 35 FVPTCs, NIFTP accounted for 20 cases (57\%), invasive EFVPTC for 12 cases (34\%), and infiltrative FVPTC for three cases (9\%). As shown in Table 6, there were no significant differences between the three subtypes in the cytological architecture and nuclear features, but the reliability is limited due to the small number of cases.

\section{DISCUSSION}

For preoperative evaluation of thyroid nodules, FNAC is a valuable and reliable method for the majority of thyroid lesions. However, FNs and similar lesions such as FVPTC or cellular $\mathrm{NH}$ are difficult to differentiate from each other using FNAC. ${ }^{3}$ For this reason, the Bethesda system proposed a flexible framework for reporting thyroid cytopathology, and the general category IV "FN or suspicious for a FN" was provided for follicular adenomas and cytomorphologically similar lesions. ${ }^{3}$ It is convenient for preoperative cytological diagnosis and can lead to a definitive diagnostic procedure, usually lobectomy, to identify carcinoma. However, the general category IV includes not only FA and FC but also hyperplastic nodules and FVPTC, ${ }^{3}$ therefore more efforts needs to be made to achieve a precise diagnosis. In the current study, we investigated 116 cases initially diagnosed as Bethesda diagnostic category IV "FN or suspicious for FN" using FNAC, and determined the cytological features that can distinguish FNs from similar lesions. Based on our observations, we suggest that trabecular patterns and cellular overlapping of the branching sheets are the most clearly characteristic cytological features of FNs, in addition to round nuclei with a granular chromatin pattern.

In this study, the FNAC-histology concordance rate for FNs was only $44 \%$, and the most common erroneous diagnosis was FVPTC (35/116 cases; 30\%), followed by NH (17/116 cases, $15 \%)$ and classic PTC (12/116 cases, 10\%). As the current study is a retrospective study of cases diagnosed by several pathologists, it has limitations of interobserver variations.

Many studies have also revealed discrepancies between the final histological and initial cytological diagnoses for cases diagnosed as FNs using FNAC, showing a concordance rate ranging from $32 \%$ to $62 \%$ (Table 7). ${ }^{6-10}$ Similar to our results, other studies reported that NH and FVPTC/PTC were most often mistaken for FNs by FNAC (Table 7). ${ }^{6-10}$ As FVPTC mimics FNs cytologically and architecturally, even in histological sections, it is commonly confused with FNs by FNAC. ${ }^{11-13}$ It might be because the nuclear changes in FVPTC can be more subtle than classic PTC. ${ }^{11,12,14}$ According to Bizzarro et al., ${ }^{12} 70 \%$ of noninvasive FVPTC cases demonstrated a nuclear size of $<20 \mu \mathrm{m}$, and rarely exhibited grooves compared to invasive FVPTCs. To overcome the limitations of morphology, the application of ancillary tests such as immunohistochemistry or molecular studies have been encouraged. ${ }^{13}$ However, these are not always available on aspirates; therefore, the cytomorphological approach is still valuable.

Recently, there has been a paradigm shift on the clinical sig- 
nificance of noninvasive EFVPTC, and nomenclature for this tumor was revised as "noninvasive follicular thyroid neoplasms with papillary-like nuclear features (NIFTP)." It is because that noninvasive EFVPTC shows indolent behavior with a very low risk of adverse outcome and therefore should not be named carcinoma. ${ }^{4}$ By adopting this new terminology, patients with NIFTP only require lobectomy and can avoid unnecessary overtreatment such as total thyroidectomy and radioactive iodine therapy., ${ }^{4} 15$

Table 6. Cytological architecture and nuclear characteristics of subtypes of FVPTC

\begin{tabular}{|c|c|c|c|c|c|c|}
\hline & $\operatorname{NIFTP}(n=20)$ & $\begin{array}{l}\text { Invasive EFVPTC } \\
\qquad(n=12)\end{array}$ & $\begin{array}{l}\text { Infiltrative FVPTC } \\
\qquad(n=3)\end{array}$ & $\begin{array}{c}p \text {-value for NIFPT } \\
\text { vs infiltrative FVPTC }\end{array}$ & $\begin{array}{l}p \text {-value for NIFPT } \\
\text { vs infiltrative }\end{array}$ & $\begin{array}{c}p \text {-value for invasive } \\
\text { EFVPTC vs infiltrative } \\
\text { FVPTC }\end{array}$ \\
\hline Microfollicular pattern & & & & .619 & .104 & .038 \\
\hline Present & $19(95)$ & $12(100)$ & $2(67)$ & & & \\
\hline Absent & $1(5)$ & $0(0)$ & $1(33)$ & & & \\
\hline Multilayer rosettes & & & & .305 & .385 & .605 \\
\hline Present & $8(40)$ & $6(50)$ & $2(67)$ & & & \\
\hline Absent & $12(60)$ & $6(50)$ & $1(33)$ & & & \\
\hline Trabecular pattern & & & & - & - & - \\
\hline Present & 0 & 0 & 0 & & & \\
\hline Absent & $20(100)$ & $12(100)$ & $3(100)$ & & & \\
\hline Branching monolayer sheets & & & & .039 & .214 & .292 \\
\hline Present & $6(30)$ & $4(33)$ & $2(67)$ & & & \\
\hline Absent & $14(70)$ & $8(67)$ & $1(33)$ & & & \\
\hline Syncytial fragments & & & & .016 & .472 & .448 \\
\hline Present & $17(85)$ & $10(83)$ & $3(100)$ & & & \\
\hline Absent & $3(15)$ & $2(17)$ & 0 & & & \\
\hline Chromatin clearing & & & & .059 & .328 & - \\
\hline Present & $15(75)$ & $12(100)$ & $3(100)$ & & & \\
\hline Absent & $5(25)$ & 0 & 0 & & & \\
\hline Nuclear groove & & & & .876 & .567 & .605 \\
\hline Present & $18(90)$ & $11(92)$ & $3(100)$ & & & \\
\hline Absent & $2(10)$ & $1(8)$ & 0 & & & \\
\hline Intranuclear cytoplasmic inclusion & & & & .465 & .484 & .792 \\
\hline Present & $11(50)$ & $5(42)$ & $1(33)$ & & & \\
\hline Absent & $9(45)$ & $7(58)$ & $2(67)$ & & & \\
\hline Anisonucleosis & & & & .876 & .567 & .605 \\
\hline Present & $18(90)$ & $11(92)$ & $3(100)$ & & & \\
\hline Absent & $2(10)$ & $1(8)$ & 0 & & & \\
\hline
\end{tabular}

Values are presented as number (\%).

FVPTC, follicular variant of papillary carcinoma; NIFTP, noninvasive follicular thyroid neoplasm with papillary-like nuclear feature; EFVPTC, encapsulated follicular variant of papillary carcinoma.

Table 7. Review of the literature of cytological-histological correlations in cases of diagnosed as follicular neoplasm in fine needle aspiration cytology

\begin{tabular}{lccccc}
\hline Histological diagnosis & Greaves et al. (2000) & Deveci et al. (2006) & Wu et al. (2012) $^{\mathbf{8}}$ & Faquin (2009) $^{9}$ & Yang et al. (2007) $^{10}$ \\
\hline FA & $25 / 96(26)$ & $145 / 339(43)$ & $35 / 65(54)$ & $88 / 251(35)$ & $109 / 326(33)$ \\
FC & $6 / 96(6)$ & $21 / 339(6)$ & $5 / 65(8)$ & $22 / 251(9)$ & $16 / 326(5)$ \\
HA & NA & NA & NA & NA & $48 / 326(15)$ \\
HC & NA & $9 / 339(3)$ & NA & NA & $13 / 326(4)$ \\
FVPTC & $6 / 96(6)$ & $36 / 339(11)$ & $7 / 65(11)$ & $40 / 251(16)$ & $71 / 326(22)$ \\
PTC & $16 / 96(17)$ & $8 / 339(2)$ & $1 / 65(1.5)$ & NA & NA \\
NH & $36 / 96(38)$ & $120 / 339(35)$ & $16 / 65(24)$ & $100 / 251(40)$ & $53 / 326(16)$ \\
HT & $6 / 96(6)$ & NA & NA & NA & $11 / 326(3)$ \\
Other malignancy & $1 / 96(1)$ & NA & NA & $1 / 251(0.3)$ & $5 / 326(2)$ \\
\hline
\end{tabular}

Values are presented as number (\%).

FA, follicular adenoma; FC, follicular carcinoma; HA, Hurthle cell adenoma; NA, not applicable; HC, Hurthle cell carcinoma; FVPTC, follicular variant of papillary thyroid carcinoma; PTC, classic papillary thyroid carcinoma; NH, nodular hyperplasia; HT, Hashimoto thyroiditis. 
For the cytological diagnosis regarding a new entity, NIFTP, more experience and consensus about NIFTP needs to be achieved for practical application. ${ }^{15}$ In case of NIFTP, there would be no clinical problem even if it is not distinguished from FA in the preoperative diagnosis, because both will be treated with lobectomy. However, in this study, there were no significant differences on cytological findings between NIFTP and invasive FVPTC. Similar results have been recently reported on cytomorphological distinction between non-invasive and invasive EFVPTC. ${ }^{16}$ In this context, it is imperative to make efforts to better differentiate FVPTC from FNs by FNAC because invasive EFVPTC and NIFTP cannot be reliably differentiated preoperatively.

As we selected the cases diagnosed as or suspected to be FNs by FNAC for the current study, all cases included in this study had moderate to high cellularity, frequently showing a wellknown microfollicular pattern. ${ }^{3}$ Usually, most PTCs contain some follicular structures in addition to conventional papillary configurations: ${ }^{17}$ therefore, it is not surprising that microfollicular patterns were identified in the aspirates of PTC and FVPTC. Furthermore, a rosette appearance with small droplets of central dense colloids was also observed not only in FNs, but also in FVPTC/PTC or NH in this study. Thus, microfollicular patterns and multilayer rosettes, which are generally accepted to be cytological characteristics of FNs, do not seem to be important in distinguishing FNs from mimicking lesions. Interestingly, the trabecular pattern was only found in the aspirates of $\mathrm{FNs}$, although the positive rates were limited to $33 \%$ and $35 \%$ of $\mathrm{FA}$ and FC cases, respectively. The trabecular pattern differs from the microfollicular pattern and multilayer rosettes in that it has a branching pattern. FNs have a three-dimensional multilayered branching structure, which could be distinguished from the monolayer appearance of the branching sheets of FVPTC (Fig. 3). When distinguishing between $\mathrm{NH}$ and FNs, some multilayered cellular ball clusters in $\mathrm{NH}$ differed from the trabecular pattern of FNs in that no branching pattern was found. Therefore, the trabecular pattern was identified as a specific feature for the differential diagnosis of FNs from mimicking lesions. Regarding cytological architecture, microfollicular patterns should no longer be considered important in the cytological diagnosis of FNs. Rather, trabecular clusters and multilayered patterns of branching cellular groups are more suitable for the proper diagnosis of FNs.

Based on our observations, the nuclear chromatin pattern and nuclear shape are considered to be important nuclear features for differential diagnosis. Because nuclear grooves were observed in NH or FNs as well as PTC, and because intranuclear inclusions were visible in less than half of the FVPTC/PTC cases in this series, there was a limit to this method of distinction. On the other hand, the nuclear chromatin of PTC/FVPTC showed ground-glass or vesicular patterns, whereas that of FNs was finely granular and $\mathrm{NH}$ showed dense chromatin. Our results are supported by a recent report on the analysis of nuclear characteristics using nuclear morphometry and textural analysis by Deka $e t$ $a l^{18}$ They reported that PTC cases showed the largest perimeter and elongation factor and heterogeneous chromatin distribution compared to FNs and NH. However, it is not easy to distinguish the nuclear chromatin pattern in each case, because FVPTC lacks the unequivocal nuclear features of PTC; this may prevent a definitive diagnosis. ${ }^{19-23}$ In addition, slightly elongated nuclei were found in FVPTC/PTC, but not in FNs or NH, suggesting that the presence of elongated nuclei in the aspirates would be helpful to differentiate PTC/FVPTC from FNs or NH. Thus, the presence of slightly elongated nuclei with chromatin clearing favors FVPTC/PTC over FNs.

In $\mathrm{NH}$, follicular cells may form large monolayers that fold over on themselves. However, they are contrasted that FNs have three-dimensional arrangement and the branching patterns. The large cell groups of $\mathrm{NH}$ did not exhibit branching patterns unlike FNs. ${ }^{10}$ Although the nuclear features were similar to those of FNs, as they showed granular chromatin patterns and occasional nuclear grooves, the nuclei of $\mathrm{NH}$ cells were slightly more hyperchromatic than those of FNs cells. Hence, we suggest that cytoarchitectural patterns of cell groups should be the main focus of differential diagnosis between $\mathrm{FNs}$ and $\mathrm{NH}$.

In conclusion, the findings of the current study suggest that a combination of architectural features and nuclear characteristics may improve the ability of cytopathologists to differentiate FNs from similar thyroid lesions such as FVPTC and NH. Based on our data, we propose that pathologists should keep in mind that the most well-known cytological feature of FNs - the microfollicular pattern with high cellularity - is not a reliable factor in obtaining a definite diagnosis. Rather, the trabecular pattern and cell clusters with cellular overlapping should be examined, as they seem to be more specific to FNs. Additionally, if slightly elongated follicular cells and nuclear clearing are found, even in a small proportion of the cells, pathologists must raise the possibility of FVPTC/PTC.

\section{Conflicts of Interest}

No potential conflict of interest relevant to this article was reported. 


\section{Acknowledgments}

This study was supported by a grant from the Korea Institute of Radiological and Medical Science (KIRAMS), funded by the Ministry of Science, ICT and Future planning, republic of Korea $(1711045543 ; 1711045540 / 50476-2017)$.

\section{REFERENCES}

1. Hamberger B, Gharib H, Melton LJ 3rd, Goellner JR, Zinsmeister AR. Fine-needle aspiration biopsy of thyroid nodules. Impact on thyroid practice and cost of care. Am J Med 1982; 73: 381-4.

2. Yassa L, Cibas ES, Benson CB, et al. Long-term assessment of a multidisciplinary approach to thyroid nodule diagnostic evaluation. Cancer 2007; 111: 508-16.

3. Cibas ES, Ali SZ. The Bethesda System for Reporting Thyroid Cytopathology. Thyroid 2009; 19: 1159-65.

4. Nikiforov YE, Seethala RR, Tallini G, et al. Nomenclature revision for encapsulated follicular variant of papillary thyroid carcinoma: a paradigm shift to reduce overtreatment of indolent tumors. JAMA Oncol 2016; 2: 1023-9.

5. Liu J, Singh B, Tallini G, et al. Follicular variant of papillary thyroid carcinoma: a clinicopathologic study of a problematic entity. Cancer 2006; 107: 1255-64.

6. Greaves TS, Olvera M, Florentine BD, et al. Follicular lesions of thyroid: a 5-year fine-needle aspiration experience. Cancer 2000; 90: $335-41$.

7. Deveci MS, Deveci G, LiVolsi VA, Baloch ZW. Fine-needle aspiration of follicular lesions of the thyroid: diagnosis and follow-up. Cytojournal 2006; 3: 9 .

8. $\mathrm{Wu} \mathrm{HH}$, Rose C, Elsheikh TM. The Bethesda system for reporting thyroid cytopathology: An experience of 1,382 cases in a community practice setting with the implication for risk of neoplasm and risk of malignancy. Diagn Cytopathol 2012; 40: 399-403.

9. Faquin WC. Diagnosis and reporting of follicular-patterned thyroid lesions by fine needle aspiration. Head Neck Pathol 2009; 3: 82-5.

10. Yang J, Schnadig V, Logrono R, Wasserman PG. Fine-needle aspiration of thyroid nodules: a study of 4703 patients with histologic and clinical correlations. Cancer 2007; 111: 306-15.

11. Brandler TC, Zhou F, Liu CZ, et al. Can noninvasive follicular thyroid neoplasm with papillary-like nuclear features be distinguished from classic papillary thyroid carcinoma and follicular adenomas by fine-needle aspiration? Cancer Cytopathol 2017; 125: 378-88.

12. Bizzarro T, Martini M, Capodimonti S, et al. Young investigator challenge: the morphologic analysis of noninvasive follicular thyroid neoplasm with papillary-like nuclear features on liquid-based cytology: Some insights into their identification. Cancer Cytopathol 2016; 124: 699-710.

13. Rossi ED, Bizzarro T, Martini M, Larocca LM, Schmitt F, Vielh P. Cytopathology of follicular cell nodules. Adv Anat Pathol 2017; 24: 45-55.

14. Clark DP, Faquin WC. Thyroid cytopathology. New York: Springer, $2005 ; 131$.

15. Krane JF, Alexander EK, Cibas ES, Barletta JA. Coming to terms with NIFTP: a provisional approach for cytologists. Cancer Cytopathol 2016; 124: 767-72.

16. Maletta F, Massa F, Torregrossa L, et al. Cytological features of "noninvasive follicular thyroid neoplasm with papillary-like nuclear features" and their correlation with tumor histology. Hum Pathol 2016; 54: 134-42.

17. Yoo C, Choi HJ, Im S, et al. Fine needle aspiration cytology of thyroid follicular neoplasm: cytohistologic correlation and accuracy. Korean J Pathol 2013; 47: 61-6.

18. Deka L, Gupta S, Gupta R, Gupta K, Kaur CJ, Singh SS. Nuclear morphometry and texture analysis on cytological smears of thyroid neoplasms: a study of 50 cases. Malays J Pathol 2017; 39: 33-7.

19. Ho AS, Sarti EE, Jain KS, et al. Malignancy rate in thyroid nodules classified as Bethesda category III (AUS/FLUS). Thyroid 2014; 24: 832-9.

20. Song JY, Chu YC, Kim L, Park IS, Han JY, Kim JM. Reclassifying formerly indeterminate thyroid FNAs using the Bethesda system reduces the number of inconclusive cases. Acta Cytol 2012; 56: 122-9.

21. Shi Y, Ding X, Klein M, et al. Thyroid fine-needle aspiration with atypia of undetermined significance: a necessary or optional category? Cancer 2009; 117: 298-304.

22. Horne MJ, Chhieng DC, Theoharis C, et al. Thyroid follicular lesion of undetermined significance: Evaluation of the risk of malignancy using the two-tier sub-classification. Diagn Cytopathol 2012; 40: 410-5.

23. Dincer N, Balci S, Yazgan A, et al. Follow-up of atypia and follicular lesions of undetermined significance in thyroid fine needle aspiration cytology. Cytopathology 2013; 24: 385-90. 